\title{
THE NOVELTY PERSPECTIVES FRAMEWORK: A NEW CONCEPTUALISATION OF NOVELTY FOR COGNITIVE DESIGN STUDIES
}

\author{
Hay, Laura; Duffy, Alex; Grealy, Madeleine \\ University of Strathclyde
}

\begin{abstract}
Novelty can be evaluated from the perspective of the designer who creates a concept (personal novelty), and people who perceive it post-creation (socio-novelty). In each case, the extent to which the concept is new compared to known artefacts is judged. The designer's evaluation is based on the same knowledge from which the concept was produced. Thus, if the concept is novel to the designer, creative cognitive processing must have occurred, i.e. something new was created in the mind. Evaluations made by other people are based on their own knowledge, which may differ from the designer's. Thus, concepts they view as novel are not necessarily the output of creative cognition. In this paper, we posit that personal novelty is directly related to designer cognition, whilst socio-novelty is not. However, existing metrics focus on the latter, and may be misleading in cognitive studies. To stimulate discussion, we formalise personal and socio-novelty in the Novelty Perspectives Framework. Empirical data suggests that the perspectives may be distinguished in practice. Future implications of the NPF are explored, highlighting the potential for insights at both the cognitive and neural level.
\end{abstract}

Keywords: Design cognition, Creativity, Evaluation

\section{Contact:}

Hay, Laura

University of Strathclyde

Design, Manufacture and Engineering Management

United Kingdom

laura.hay@strath.ac.uk

Cite this article: Hay, L., Duffy, A., Grealy, M. (2019) 'The Novelty Perspectives Framework: A New Conceptualisation of Novelty for Cognitive Design Studies', in Proceedings of the 22nd International Conference on Engineering Design (ICED19), Delft, The Netherlands, 5-8 August 2019. DOI:10.1017/dsi.2019.42 


\section{INTRODUCTION}

Two approaches may be applied to gain a view on the cognitive mechanisms involved in creative design ideation: (1) protocol analysis, where the designer verbally reports their processing and inferences are made about cognition based on the resulting protocols; and (2) output-based experiments, where performance metrics linked to some aspect of processing are evaluated and observations are made about cognition based on the results (Hay et al., 2017a). The former approach has been applied prolifically since the inception of design research in the late 1960s, but has been criticised for its subjectivity and unsuitability for studying large samples. Accordingly, controlled output-based approaches, which are characteristic of the behavioural experiments conducted by cognitive psychologists, have seen increasing application in recent years (Dinar et al., 2015).

A key challenge for output-based cognitive studies is the identification of appropriate creative performance metrics, with novelty positioned as fundamentally important in this respect. We interpret novelty as referring to newness (Chakrabarti and Khadilkar, 2003), although alternative definitions exist (Brown, 2014). The novelty of a design concept may be considered from two perspectives, i.e. that of: (i) the designer who creates it; and (ii) people who perceive and interpret it after it has been created (Simonton, 2017). In each case, novelty is evaluated relative to the assessor's artefact knowledge - that is, a judgment is made about the extent to which a proposed concept is new compared to known existing artefacts. Clearly, different people may have different artefact knowledge and therefore produce different novelty evaluations (Kaufman et al., 2008). However, a key distinction between the designer and other people is that for the former, evaluation is based on the same source of knowledge from which the concept was produced. In this respect, Benedek et al. (2013, p.125) note that ideation typically produces two kinds of concept: those "newly created during the task" and those "recalled from memory." It may be seen that if the concept has a degree of novelty to the designer, some kind of creative processing must have occurred during ideation - something new was created in the mind of the designer. Conversely, if there is no novelty to the designer, then creation cannot have occurred - it is likely that the concept was simply retrieved from memory.

Based on the above, it can be posited that a designer's personal evaluations of novelty are directly related to their creative cognitive processing. In contrast, it appears that evaluations of novelty from the perspective of other people - which we may term 'socio-novelty' - are not. A concept that is perceived as novel by people independent of the designer is not necessarily the output of creative cognitive processes, and vice versa. Relying solely on metrics of socio-novelty in cognitive studies of design ideation may therefore provide a misleading view on designer cognition, which could be remedied by the inclusion of personal novelty metrics. However, these two perspectives on novelty do not appear to be clearly elucidated in the existing literature, and tend not to be considered by authors proposing and selecting metrics for novelty evaluation in cognitive design studies.

To provide clarity and a basis for advancing robust cognitive research on creative design ideation, in this paper we propose and position a new conceptualisation of design novelty: the Novelty Perspectives Framework (NPF). The purpose of this work is primarily to stimulate a discussion on the way that we conceptualise design novelty, and the effects this may have on cognitive design studies. Building on the existing literature on novelty, the NPF formalises and relates the perspectives of personal novelty and socio-novelty for the first time and describes the associated evaluation processes. The framework suggests that although personal and socio-novelty evaluations may match in certain cases, only the former seem to directly and reliably provide information on the nature of the designer's internal ideation processes. We map existing socio-novelty evaluation methods to the framework, and discuss the need for measures of personal novelty in design. A comparison of personal and socio-novelty evaluation, using results from a recent study of creative design ideation, suggests that the two perspectives may be distinguished in practice. However, further research is needed to build upon these initial findings. We conclude by discussing the implications of the NPF for future cognitive studies of design ideation.

\section{THE NOVELTY PERSPECTIVES FRAMEWORK}

\subsection{The role of novelty evaluation in creative design ideation}

Several models and theoretical viewpoints on creative ideation have been proposed in cognitive psychology (Finke et al., 1992), neuroscience (Beaty et al., 2016), and design cognition research (Jin 
and Chusilp, 2006). One widely discussed view - illustrated in Figure 1 - posits that the ideas created by a person are founded in their knowledge and experiences, and produced through the association, integration, and transformation of internal representations (Finke et al., 1992). These representations are retrieved from episodic (contextual) and semantic (conceptual) memory in response to retrieval cues, which may be derived from externally perceived information sources or internally generated thoughts and ideas (Liikkanen and Perttula, 2010). The solutions proposed during ideation tasks may be created anew in this way, or they may be known concepts recalled directly from memory (Benedek et al., 2013) as shown in Figure 1. To illustrate in the context of design: upon reading a design brief (retrieval cue), a designer may recall knowledge about a similar problem they have previously worked on (episodic memory) and/or knowledge about types of functions and mechanisms (semantic memory) that may be useful for addressing the problem. They may then form associations between the representations, integrate key properties from each one to create a new artefact concept, and execute a transformation to evolve the emergent concept. Alternatively, if they have worked on the problem in the past, they may start by directly recalling a known past solution from memory.

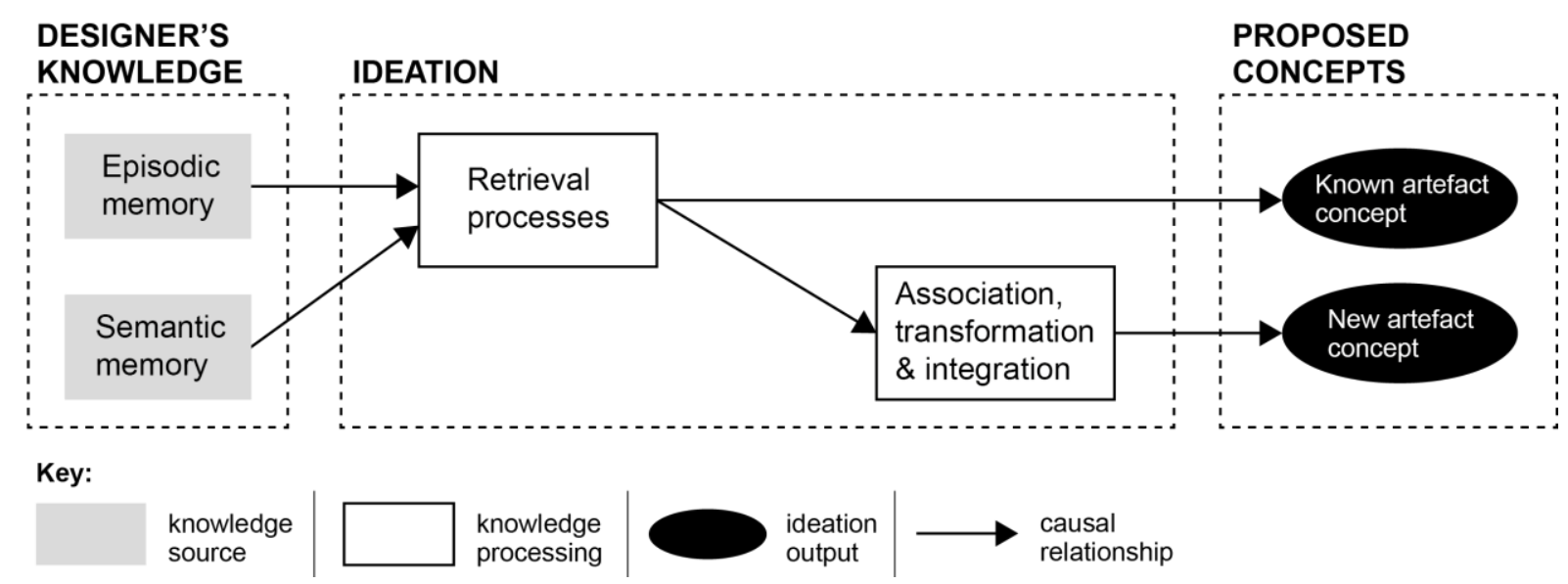

Figure 1: Creation and recall of concepts during ideation

Beaty et al. (2016, p.92) suggest that processing during ideation is constrained and directed "to meet task-specific goals via top-down monitoring and executive control." A key process involved in this top-down regulation is the evaluation of ideas to determine whether they satisfy the goals of the task. Whilst a designer may consider numerous goals, the production of novel solutions to a given design problem may be viewed as a fundamental goal of creative design ideation. Thus, the evaluation of concept novelty can be considered to constitute a basic component of executive processing in this context (Hay et al., 2017b).

As well as the regulatory role that novelty evaluation plays during ideation, ideation outputs are also evaluated by people independent of the designer after they have been proposed. In an empirical context, this kind of independent evaluation is commonly conducted by experimenters and/or design experts, using systematic scoring methods or opinion-based methods such as ratings (Shah et al., 2003). Here, the independence of the assessor from the designer is considered to be fundamentally important for maintaining objectivity in the evaluation, and ensuring that the results are not biased by the designer's perspective on their own designs. However, we propose that in cognitive studies, the designer's personal perspective on novelty may provide more reliable information on their internal ideation processes than evaluations made by independent assessors. This position is elaborated in the sub-sections below.

\subsection{Personal and socio-novelty}

In general terms, evaluation is the process of assessing the relation between an entity and some kind of reference with respect to a criterion/set of criteria (Duffy and O'Donnell, 1998). The evaluation of novelty in design ideation involves assessing the extent to which a proposed artefact concept is new relative to some known set of artefacts (Chakrabarti and Khadilkar, 2003; Shah et al., 2003). When a designer evaluates their own concepts, they compare these with related artefacts that they know of (the reference). Based on this comparison, they formulate judgments about the extent to which their concepts are new. The knowledge sources supporting this evaluation are the same as those driving the production of concepts in Figure 1, i.e. the designer's episodic and semantic memory. 
When an agent independent of the designer (e.g. an experimenter) evaluates a concept proposed during ideation, the process differs somewhat from the above. Firstly, during ideation tasks, designers typically express their proposed concepts in rough sketches and textual descriptions. Thus, it is necessary for the agent to identify the concept from these representations in order to evaluate it. Secondly, the agent compares the identified concept with related artefacts that they know of in order to formulate a novelty judgment. In this case, novelty evaluation is based on the knowledge of an independent agent, which may not necessarily match the designer's artefact knowledge (from which the concept was created).

Based on the above, two perspectives on novelty evaluation may be described in the context of design ideation (formalised in Figure 2): (i) personal novelty, where the designer evaluates the concepts they produce by comparing against their own artefact knowledge; and (ii) socio-novelty, where an independent agent evaluates concepts - identified from representations produced by a designer - via comparison against their own artefact knowledge. Personal novelty judgments are based on the same sources of knowledge from which a concept was produced, and socio-novelty judgments are based on independently held knowledge. The knowledge of each individual may be viewed as a subset of the total artefact knowledge in society, i.e. all that could conceivably be known by the involved humans about designed artefacts. The processes involved in evaluating personal and socio-novelty are formalised and related in the Novelty Perspectives Framework (NPF), presented in Figure 2.

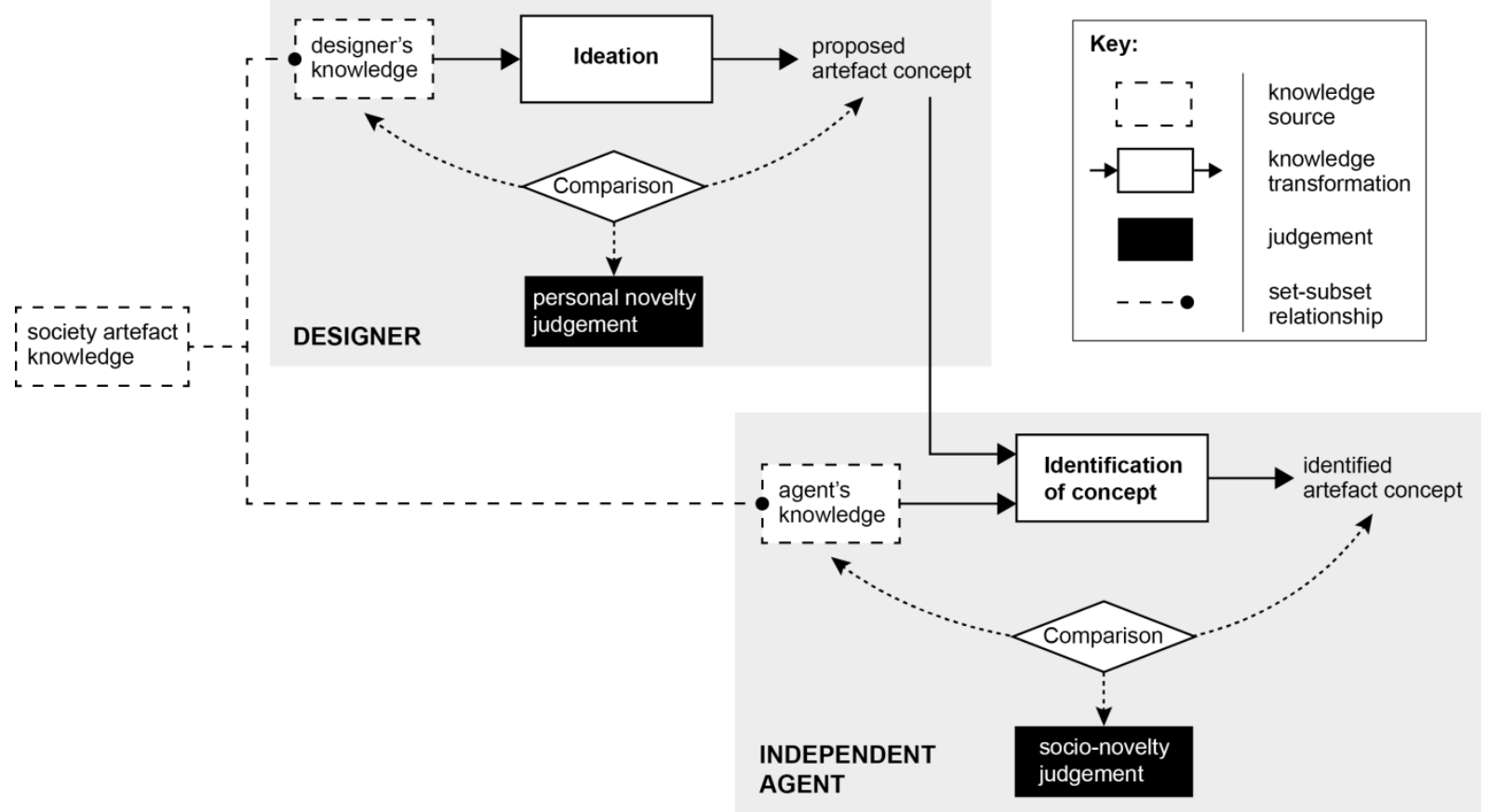

Figure 2: The novelty perspectives framework (NPF)

The evaluation of socio-novelty is well established in design research. The following three methods are frequently applied (with various adaptations) to assess and analyse design ideation (Shah et al., 2003), and may all be mapped to the evaluation of socio-novelty in the NPF:

- Method 1: Proposed concepts are compared against pre-selected reference artefacts, which have been rated according to how novel an experimenter considers them to be as solutions to the design problem. A concept is assigned the score of its most closely matching reference artefact.

- Method 2: The novelty of proposed concepts is rated by an experimenter relative to reference artefacts that they implicitly select during evaluation (i.e. the reference is not pre-specified).

- Method 3: The total sample of concepts produced by designers in a study is interpreted by an experimenter to identify the range of different artefact types proposed. The novelty of any given artefact concept is then computed as the infrequency with which that particular type of artefact appears in the broader sample of concepts.

In all three methods, it is necessary for the experimenter to firstly identify concepts from the representations produced by designers (e.g. sketches and models) to evaluate them, as shown in the NPF. Furthermore, the reference against which identified concepts are compared to judge novelty 
always consists of some component of the experimenter's artefact knowledge, e.g. explicitly preselected artefacts in method 1, and artefacts implicitly selected during evaluation in method 2 . This is true even in the case of method 3, which is systematic rather than opinion-based: to calculate infrequency, the experimenter must compare one identified concept against the range of artefacts they identified from the sample.

In comparison with socio-novelty, the evaluation of personal novelty does not appear to be explicitly discussed at any length in the design literature. This is a notable gap in the context of cognitive studies, because - as discussed further below - it is personal novelty rather than socio-novelty that appears to be directly related to a designer's creative cognitive processing.

\subsection{Personal novelty as an indicator of cognitive processing}

The difference between personal novelty and socio-novelty as outlined in Section 2.2 is reflected in the work of Benedek et al. $(2013,2018)$, who report neurocognitive studies on creative ideation in the general population. The authors state that whilst ideas may be viewed as 'creative' in some dimension by external observers, this "does not necessarily imply that these ideas are the result of a genuinely creative process." Only ideas that are new to the ideator are "the result of a genuinely creative act in which previously unrelated frames of thought become associated in a new and meaningful way" (Benedek et al., 2013, p.126). The authors study the Alternate Uses Task using fMRI, where subjects are asked to generate original uses for common objects such as a shoe, a hat, etc. In order to distinguish periods of creation from periods of recall, subjects were asked post-MRI scan to indicate whether the original uses they proposed were newly created or recalled from memory. The fMRI results suggest that the creation of new concepts is differentiated from recall at the neural level by increased activation in brain regions associated with: (1) the integration of information in new ways; and (2) executively controlled mental simulation (Benedek et al., 2013, 2018).

In the above fMRI studies, concepts self-reported as newly created were found to be associated with increased activation in brain regions supporting particular cognitive processes. This suggests that evaluations of personal novelty - that is, novelty as self-reported by the designer - may be directly related to variations in mental processing during ideation. This is conveyed by the NPF introduced in Section 2.2 (Figure 2). Zero novelty seems to indicate that a proposed concept was already known to the designer, and implies that they engaged in direct recall from long term memory rather than creation (Figure 1). A degree of novelty appears to indicate that the proposed concept, or part thereof, was created anew during the ideation task through some kind of associative/transformative/integrative processing.

In contrast, it is questionable whether socio-novelty judgments made by independent agents have any direct relationship with the designer's creative cognitive processing. They are based on an interpretation of the proposed artefact concept derived from representations such as sketches, which may not match the intention of the designer. More fundamentally, the identified concept is compared against the agent's artefact knowledge, which may not match the knowledge of the designer who proposed it. Thus, it is possible that a concept could be judged as highly novel by an independent agent, but was already known by the designer - and vice versa. In this situation, metrics focusing on socio-novelty would provide misleading information on the designer's cognition: it may appear that they engaged in the creation of a new concept, when in reality they recalled a known artefact from memory (and vice versa). Evaluations of socio-novelty reflect an independent agent's internal processing and knowledge rather than that of the designer. From this viewpoint, it seems that metrics focusing on personal novelty are needed in addition to those measuring socio-novelty in cognitive studies. Although the two evaluations may match in certain cases, only the former seems to directly reflect the designer's creative cognition.

\section{COMPARING PERSONAL AND SOCIO-NOVELTY EVALUATION}

As noted in Section 1, the purpose of this paper is primarily to stimulate a discussion on the way that we conceptualise design novelty, and the effects this may have on cognitive design studies. As such, we do not claim to be able to provide conclusive evidence on the nature of personal and socio-novelty evaluation as formalised in the NPF, or concrete recommendations for measures and methods. However, we offer some initial observations for future consideration and exploration from a recent study on cognition in creative design ideation, where we compared the results of personal and socionovelty evaluation methods. 


\subsection{Study details}

The study aimed to investigate differences in the cognitive processing of product design engineers during two types of design ideation task, where they were asked to generate solutions to:

- Creative (i.e. open-ended) problems, e.g.: "Lighting towns and cities at night has negative environmental impacts e.g. fossil fuel depletion, light pollution, and disruption to wildlife. Generate concepts for products to improve the environmental impacts of lighting urban areas."

- Innovative (i.e. specifying a desired solution type) problems, e.g.: "Street lighting powered through the National Grid creates high annual running costs and negative environmental impacts for local authorities. Generate concepts for a self-powered street light that does not use mains electricity."

A sample of 30 professional product design engineers participated in the study (cumulative industrial experience $=225$ years, mean $=7.5$ years, $\mathrm{SD}=7.2$ ). Each designer completed ten creative and ten innovative design tasks. Each designer's tasks were randomly selected from a larger pool of forty tasks (twenty of each type) and presented in a random order during the study. Each designer produced a maximum of three concepts per task, and externalised these in rough annotated sketches (see Figure 3 ). The findings from the analysis of cognitive processing are not the focus of this paper, but in Section 3.3 we compare personal and socio-novelty evaluation results obtained for a subset of 22 participants from whom we were able to gather personal novelty ratings. The evaluation methods used are outlined in Section 3.2.

\subsection{Evaluation methods}

By their nature, personal novelty judgments must be self-reported. One way to gather this kind of data is through the use of a rating scale. In our study, we asked the designers to rate the novelty of their own design concepts ( 60 per designer) on a scale from 0 (not novel) to 9 (very novel). Another consideration is whether the novelty ratings should be provided immediately after each concept is generated (concurrently), or retrospectively at the end of the ideation session. It seems that the former is likely to interfere with the ideation process and the novelty of the concepts produced, e.g. through the phenomenon of evaluation apprehension (Finke et al., 1992). In our study, we asked designers to rate personal novelty retrospectively by reviewing their concept sketches after completion of all twenty tasks. All designers were provided with an identical set of rating instructions and response sheets, and were able to provide ratings for the majority of their concepts.

A weakness in our approach to evaluating personal novelty is the lack of reliability testing. In conventional expert rating approaches, reliability is typically assessed using statistical measures of inter-rater reliability. These measures quantify the extent to which independent raters agree on the ratings assigned to concepts using the same scale (Srivathsavai et al., 2010). This kind of reliability is not applicable in the case of personal novelty, because each rater (i.e. designer) evaluates a different set of concepts (i.e. their own). An alternative approach is to measure intra-rater reliability, i.e. the extent of agreement between the ratings provided by a single rater for the same set of concepts at different times. We could have asked the designers in our study to rate a subset of their concepts again at a later date using the same scale, and compared these with their original ratings to determine the extent of agreement. However, this was not achievable due to constraints on the time that designers could commit to the study.

To measure socio-novelty, we applied Method 3 outlined in Section 2.2 (sample infrequency). Briefly, the following procedure was undertaken in the context of each design task, adapted from the work of Shah et al. (2003) and its numerous derivatives (e.g. Fiorineschi et al., 2018; Nelson et al., 2009; Sluis-Thiescheffer et al., 2016):

1. The range of solutions proposed by the sample of designers was firstly identified from concept sketches using a qualitative coding approach. This involved interpreting each individual sketch to identify the type of solution generated by the designer (e.g. Figure 3). Over the course of this process, a set of codes describing the range of solution types identifiable across the full set of sketches produced for the task was defined (e.g. Table 1). Sketches were initially coded by one design researcher; inter-coder reliability was tested using a further two coders (a design professional and a $\mathrm{PhD}$ student in design cognition) who independently coded $\sim 16 \%$ of the total concept sample. Acceptable reliability was achieved (Krippendorff's alpha $=0.79$ ). 
2. The novelty of each identified solution type was computed as the infrequency with which it appears in the set of concept sketches produced for the task, as per Equation 1:

Solution novelty $=1-\frac{\text { no.of sketches coded with solution }}{\text { total no.of sketches produced }}$

For example, 67 sketches were produced and coded for the task referenced in Table 1 . The novelty score of the solution coded in Figure 3, which was identified in 2 sketches as per Table 1, is therefore 0.970. Each sketched concept was then assigned the novelty score associated with its coded solution type. For instance, the concept presented in Figure 3 would be assigned a novelty score of 0.970 .

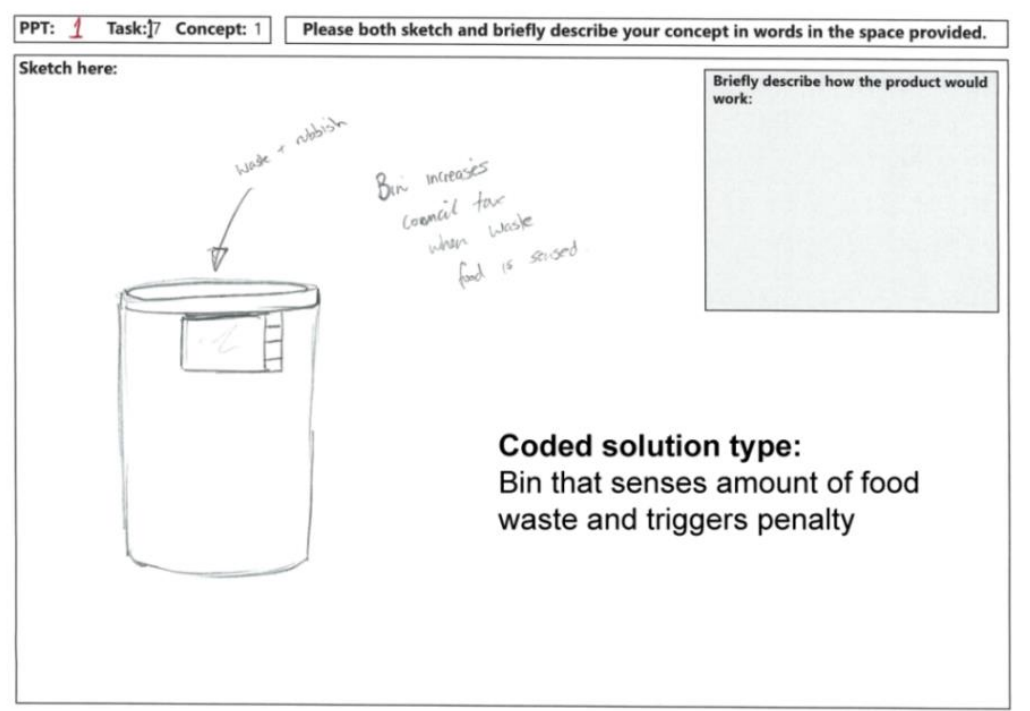

Figure 3. Example of a sketch coded with a solution type

Table 1. Set of codes developed for task focused on reducing domestic food waste

\begin{tabular}{|l|l|}
\hline Code description & No. of sketches coded \\
\hline System that transforms food waste into fertiliser & 9 \\
\hline App that uses info on stored food to generate/suggest recipes & 8 \\
\hline Fridge that notifies user about expiry & 7 \\
\hline Device that stores food in a vacuum & 6 \\
\hline Device that controls/maintains food storage temperature & 4 \\
\hline Box that preserves leftover/unwanted food for longer & 3 \\
\hline Food processor that turns leftovers into different food types & 3 \\
\hline Bin that senses amount of food waste \& triggers penalty & 2 \\
\hline Date tracking app for mobile device & 2 \\
\hline Food packaging/labels that indicate expiry & 2 \\
\hline Portioning phone app & 2 \\
\hline System for growing own vegetables at home & 2 \\
\hline System that delivers food on demand/as needed from shop & 2 \\
\hline Transparent fridge door & 2 \\
\hline App that provides info on how to reuse food waste as pet food & 1 \\
\hline Bin that indicates amount of food wasted over time & 1 \\
\hline Customisable food packages/portions & 1 \\
\hline Freeze-dried food portions & 1 \\
\hline Fridge compartments that indicate what food should be used first & 1 \\
\hline Fridge that generates aroma to stimulate appetite & 1 \\
\hline Hunger sensor & 1 \\
\hline Multi-portion food packaging & 1 \\
\hline
\end{tabular}




\begin{tabular}{|l|l|}
\hline Portion scales & 1 \\
\hline Recipe cards & 1 \\
\hline Shelving that controls order in which stored food is accessed & 1 \\
\hline $\begin{array}{l}\text { System that uses info on stored food to generate shopping } \\
\text { requirements }\end{array}$ & 1 \\
\hline $\begin{array}{l}\text { Tube network that delivers unwanted food from one household to } \\
\text { another }\end{array}$ & 1 \\
\hline
\end{tabular}

\subsection{Results}

A total of 1158 concepts were generated by the 22 participants included in the comparison and evaluated. Table 2 presents summary statistics for the personal and socio-novelty scores obtained via the above methods. It may be seen from visual inspection of the scatterplot in Figure 4 that there is no correlation between the two metrics. This suggests that a distinction may indeed be made between personal and socio-novelty in practice - that is, the metrics appear to measure fundamentally different dimensions. However, further studies using different types of metric are needed to build on these initial findings. In this respect, it should be noted that the evaluation reference used in the socionovelty metric applied here (knowledge of artefacts identified from a sample of concepts generated by study participants) is not directly comparable with the reference used in the personal novelty ratings (the designer's general artefact knowledge). The comparison of designer novelty ratings with ratings provided by independent experts may be more appropriate, and could provide greater insight into the nature of personal and socio-novelty and any relationship between them. Furthermore, the results reported here clearly do not provide any insight into the possible relationship between personal novelty and a designer's creative cognition as postulated in this paper. At the very least, the results highlight that the use of personal and socio-novelty metrics in a study may produce fundamentally different results. Decisions about which type of metric to use may therefore warrant deeper consideration than they seem to be given at present.

Figure 4. Scatterplot of socio novelty against personal novelty

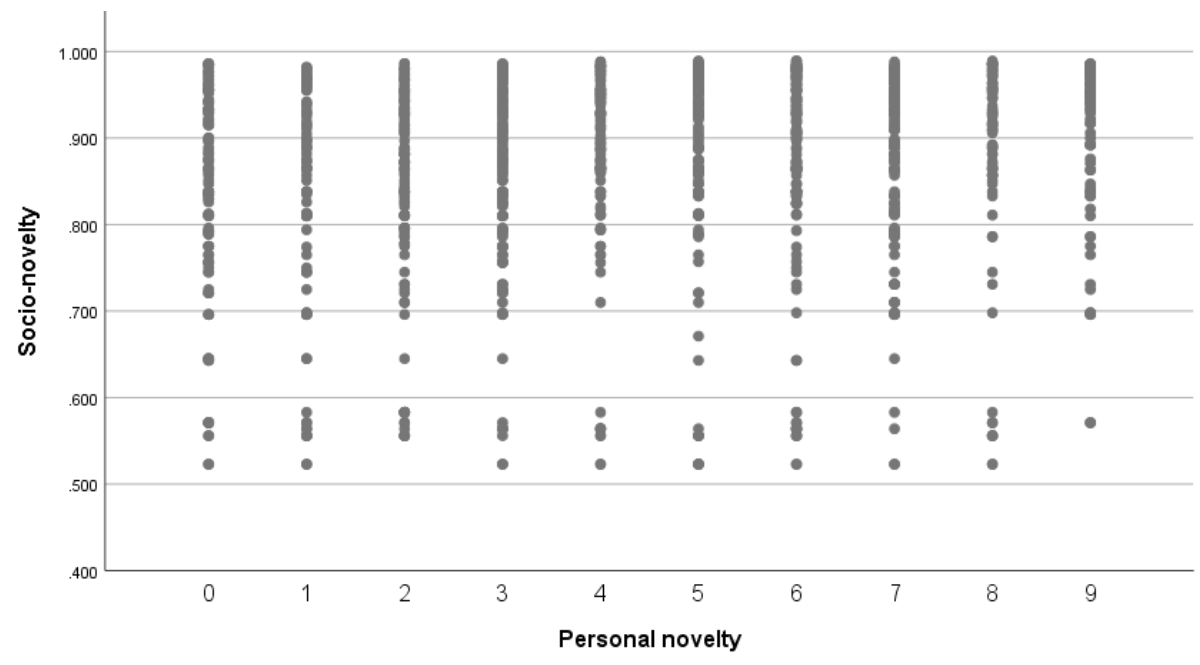

Table 2. Summary statistics for personal and socio-novelty scores obtained for concept sketches $(n=1158)$

\begin{tabular}{|l|l|l|}
\hline & Personal novelty & Socio-novelty \\
\hline Range & $0-9(9)$ & $0.523-0.989(0.466)$ \\
\hline Mean & 4.33 & 0.863 \\
\hline Median & 4.00 & 0.893 \\
\hline Mode & 2.00 & 0.838 \\
\hline Standard deviation & 2.72 & 0.118 \\
\hline Skew & 0.02 & -1.34 \\
\hline
\end{tabular}




\section{CONCLUSION AND FUTURE IMPLICATIONS}

In this paper, we have presented a new conceptualisation of design novelty: the Novelty Perspectives Framework (NPF). The NPF describes the evaluation of a design concept's novelty through comparison with known artefacts. Two perspectives on novelty are formalised in this respect: (i) personal novelty, where the designer compares their concepts with their own artefact knowledge; and (ii) socio-novelty, where an independent agent identifies proposed concepts from representations produced by the designer, and compares them with their own artefact knowledge. As discussed in Section 2, the NPF suggests that personal novelty is directly related to a designer's creative cognitive processing during ideation: if the concepts produced have a degree of novelty to the designer, this implies that something new was created through associative, transformative, and/or integrative processing of internal representations from episodic and semantic memory. If there is no novelty to the designer, the concepts were likely recalled directly from memory (i.e. already known). This is supported to some extent by recent neuroimaging work on creative ideation in the general population. In contrast, socio-novelty does not necessarily reflect the designer's cognition. Since the designer and an independent agent may have different artefact knowledge, and different interpretations of the proposed concept, it is possible that a concept viewed as highly novel by the latter was already known to the designer and vice versa. Thus, metrics of socio-novelty do not appear to be a reliable indicator of a designer's creative cognitive processing. The comparison of personal and socio-evaluation presented in Section 3 suggests that the two perspectives may be distinguished in practice, and that applying metrics of personal and socio-novelty in studies may produce fundamentally different results. However, further research is needed to build upon these initial findings, and to investigate the potential relationship between personal novelty and a designer's creative cognitive processing.

With the above limitations noted, the observations on personal and socio-novelty highlighted by the NPF nonetheless have implications for the validity of results in cognitive and neurocognitive investigations of creative design ideation. For example, say we wish to study the effect of examples on creative cognitive processing during ideation (e.g. in studies of analogical reasoning and fixation). It seems that socio-novelty metrics alone may not be capable of providing reliable information on variations in this processing across the different experimental conditions. If we wish to investigate brain activity associated with the creation of new design concepts, we need a measure that we would expect to covary with this activity. Again, it seems that this would not necessarily be the case with socio-novelty metrics. In both of these examples, the use of socionovelty metrics may produce misleading results and lead to inappropriate conclusions about cognition and brain activity in design ideation. However, as noted in Section 2, the socio-novelty perspective dominates existing evaluation methods in design. There is a need for the development of appropriate and reliable methods for evaluating personal novelty so that we can explore whether the two novelty perspectives can be differentiated in practice, the relationships between personal novelty and cognition proposed in the NPF, and what all of this means for the robustness of cognitive design research.

Methods for evaluating personal novelty in design would also open up avenues for design ideation studies mirroring the neurocognitive work on general creative ideation tasks by Benedek et al. $(2013,2018)$. That is, studies exploring the brain regions and cognitive processes involved in the creation of new design concepts versus the recall of known concepts. This could provide deeper scientific insights into a range of phenomena already investigated extensively in design research, e.g. fixation, case-based reasoning, analogical reasoning, and design synthesis.

Finally, it should be noted that in certain contexts, socio-novelty metrics may be perfectly acceptable measures of design novelty. For instance, if the goal is to design a new product for a particular user or customer, or to produce ideas that are novel in a societal context, it would be more appropriate to focus on socio-novelty than personal novelty. It has been suggested in the literature on both design (Sosa and Gero, 2005) and general creativity (Simonton, 2017) that the novelty of concepts in a social context is the outcome of a consensus-building process between multiple agents, and can thus change over time as the consensus shifts. The involvement of multiple independent agents is not currently represented within the NPF, but could constitute a future development. We are also not suggesting that socio-novelty metrics are of no value whatsoever in cognitive studies; investigating the relationship between personal and socio-novelty in this context presents a salient line of enquiry. Our position is simply that personal novelty appears to be directly related to creative cognitive processing during ideation whilst socio-novelty is not, an observation that has been overlooked in the literature to date. We intend for this paper to serve as a basis for discussion around these ideas. 


\section{REFERENCES}

Beaty, R.E., Benedek, M., Silvia, P.J. and Schacter, D.L. (2016), "Creative Cognition and Brain Network Dynamics”. Trends Cogn. Sci., Vol. 20, pp. 87-95. https://doi.org/10.1016/J.TICS.2015.10.004

Benedek, M., Jauk, E., Fink, A., Koschutnig, K., Reishofer, G., Ebner, F. and Neubauer, A.C. (2013), “To create or to recall? Neural mechanisms underlying the generation of creative new ideas". Neuroimage Vol. 88, pp. 125-133. https://doi.org/10.1016/j.neuroimage.2013.11.021

Benedek, M., Schües, T., Beaty, R.E., Jauk, E., Koschutnig, K., Fink, A. and Neubauer, A.C. (2018), “To create or to recall original ideas: Brain processes associated with the imagination of novel object uses". Cortex Vol. 99, pp. 93-102. https://doi.org/10.1016/J.CORTEX.2017.10.024

Brown, D. (2014), Problems with the Calculation of Novelty Metrics, in: Proc. Design Creativity Workshop, 6th Int. Conf. on Design Computing and Cognition (DCC'14).

Chakrabarti, A. and Khadilkar, P. (2003), A measure for assessing product novelty. Int. Conf. Eng. Des. ICED’03.

Dinar, M., Shah, J.J., Cagan, J., Leifer, L., Linsey, J., Smith, S.M. and Hernandez, N.V., (2015), "Empirical Studies of Designer Thinking: Past, Present, and Future”. J. Mech. Des. Vol. 137, pp. 1-13. https://doi.org/10.1115/1.4029025

Duffy, A.H.B. and O’Donnell, F.J. (1998), “A Design Research Approach, in: Critical Enthusiasm Contributions to Design Science. Tapir”, Trondheim, pp. 33-40.

Finke, R.A., Ward, T.B. and Smith, S.M. (1992), Creative Cognition: Theory, Research, and Applications. The MIT Press, Cambridge, MA; London, England.

Fiorineschi, L., Frillici, F.S. and Rotini, F. (2018), Issues Related To Missing Attributes in a- Posteriori Novelty Assessments, in: International Design Conference - Design 2018. pp. 1067-1078. https://doi.org/10.21278/idc.2018.0118

Hay, L., Duffy, A.H.B., McTeague, C., Pidgeon, L.M., Vuletic, T. and Grealy, M. (2017a), “A systematic review of protocol studies on conceptual design cognition: Design as search and exploration”. Des. Sci. Vol. 3, pp. 1-36. https://doi.org/10.1017/dsj.2017.11

Hay, L., Duffy, A.H.B., McTeague, C., Pidgeon, L.M., Vuletic, T. and Grealy, M. (2017b), “Towards a shared ontology: A generic classification of cognitive processes in conceptual design”. Des. Sci. Vol. 3, pp. 1-42. https://doi.org/10.1017/dsj.2017.6

Jin, Y. and Chusilp, P. (2006), "Study of mental iteration in different design situations”. Des. Stud. Vol. 27, pp. 25-55. https://doi.org/10.1016/j.destud.2005.06.003

Kaufman, J.C., Baer, J., Cole, J.C. and Sexton*, J.D. (2008), “A Comparison of Expert and Nonexpert Raters Using the Consensual Assessment Technique”. Creat. Res. J. Vol. 20, pp. 171-178. https://doi.org/10.1080/10400410802059929

Liikkanen, L.A. and Perttula, M. (2010), "Inspiring design idea generation: insights from a memory-search perspective”. J. Eng. Des. Vol. 21, pp. 545-560. https://doi.org/10.1080/09544820802353297

Nelson, B.A., Wilson, J.O., Rosen, D. and Yen, J. (2009), "Refined metrics for measuring ideation effectiveness". Des. Stud. Vol. 30, pp. 737-743. https://doi.org/10.1016/j.destud.2009.07.002

Shah, J.J., Smith, S.M. and Vargas-Hernandez, N. (2003), "Metrics for measuring ideation effectiveness". Des. Stud. Vol. 24, pp. 111-134. https://doi.org/10.1016/S0142-694X(02)00034-0

Simonton, D.K. (2017), Big-C Versus Little-c Creativity: Definitions, Implications, and Inherent Educational Contradictions, in: Creative Contradictions in Education. Springer, Cham, pp. 3-19. https://doi.org/10.1007/978-3-319-21924-0_1

Sluis-Thiescheffer, W., Bekker, T., Eggen, B., Vermeeren, A. and De Ridder, H. (2016), "Measuring and comparing novelty for design solutions generated by young children through different design methods". Des. Stud. Vol. 43, pp. 48-73. https://doi.org/10.1016/J.DESTUD.2016.01.001

Sosa, R. and Gero, J.S. (2005), A computational study of creativity in design: The role of society. AI EDAM Vol. 19, pp. 229-244. https://doi.org/10.1017/S089006040505016X

Srivathsavai, R., Genco, N., Hölttä-Otto, K. and Seepersad, C.C. (2010), Study of Existing Metrics Used in Measurement of Ideation Effectiveness, in: Volume 5: 22nd International Conference on Design Theory and Methodology; Special Conference on Mechanical Vibration and Noise. ASME, pp. 355-366. https://doi.org/10.1115/DETC2010-28802

\section{ACKNOWLEDGMENTS}

This research was supported by the United Kingdom's Engineering and Physical Sciences Research Council, grant number EP/M012123/1. 